\title{
Bistability in Kerr lens mode-locked Ti:sapphire lasers
}

\author{
Marcelo G. Kovalsky ${ }^{\text {a,* }}$, Alejandro A. Hnilo ${ }^{\text {a }}$, Ariel Libertun ${ }^{\text {b }}$, \\ Mario C. Marconi ${ }^{b}$ \\ ${ }^{a}$ Centro de Investigaciones en Laseres y Aplicaciones (CEILAP), Instituto de Investigaciones Científicas y Técnicas de las \\ Fuerzas Armadas (CITEFA), Consejo Nacional de Investigaciones Científicas y Técnicas (CONICET), Universidad Nacional de \\ San Martín (UNSAM), Zufriategui 4380, (1603) Villa Martelli, Argentina \\ b Laboratorio de Electrónica Cuántica, Departamento de Física, Facultad de Ciencias Exactas y Naturales, Universidad de Buenos Aires, \\ Pabellon 1, Ciudad Universitaria (1429), Buenos Aires, Argentina
}

Received 12 November 2000; received in revised form 19 March 2001; accepted 19 March 2001

\begin{abstract}
Femtosecond pulse Ti:sapphire lasers can operate in different ways for the same values of the control parameters. This phenomenon of multistability is explained in a simple way by a theoretical approach using iterative or Poincaré maps. We present experimental confirmation of the predictions of the approach regarding the slope (of pulse duration vs. group velocity dispersion) and regions of stability of two different regimes of mode locking, i.e. transform-limited and chirped output pulses. (C) 2001 Elsevier Science B.V. All rights reserved.
\end{abstract}

Keywords: Self-mode-locked lasers; Nonlinear dynamics; Bifurcations and chaos; Ti:Sapphire lasers; Kerr lens mode locking

\section{Introduction}

The Kerr lens mode-locking (KLM) Ti:sapphire laser has been a cornerstone of ultrafast science since its first demonstration by Spence et al. [1]. Because of the nonlinear effects required for KLM operation, it is not surprising that lasers of this kind exhibit a variety of interesting dynamics, such as: beam breakup [2], self $Q$-switching [3], period doubling and tripling [4,5] and even chaos [6-8]. In this paper, we report the observation and theoretical description of bistable behavior between two different regimes of mode locking. The bistability occurs for a range of values of the cavity

\footnotetext{
${ }^{*}$ Corresponding author.

E-mail address: mkovalsky@citefa.gov.ar (M.G. Kovalsky).
}

group velocity dispersion (GVD), in which both regimes are stable. For other values of GVD, only one regime of mode locking is stable. The phenomena are satisfactorily described by an approach using a five-variables iterative or Poincaré map. In Section 2, we briefly review the map's approach. In Section 3, we describe the experimental setup and compare observations and predictions.

\section{Mode-locking multistability}

Even though KLM Ti:sapphire lasers are extraordinarily stable sources of fs pulses, it is often observed that the mode-locking operation disappears, and the output becomes continuous $(\mathrm{CW})$. In this case, mode locking is usually restored by 
(a)

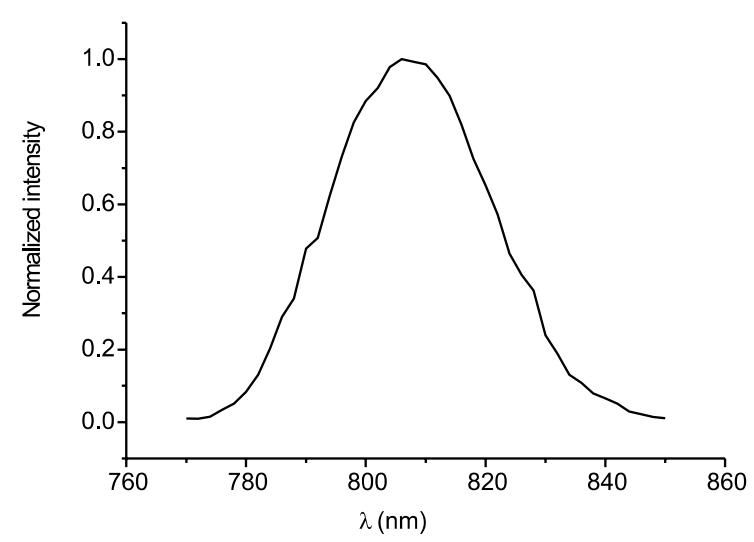

(b)

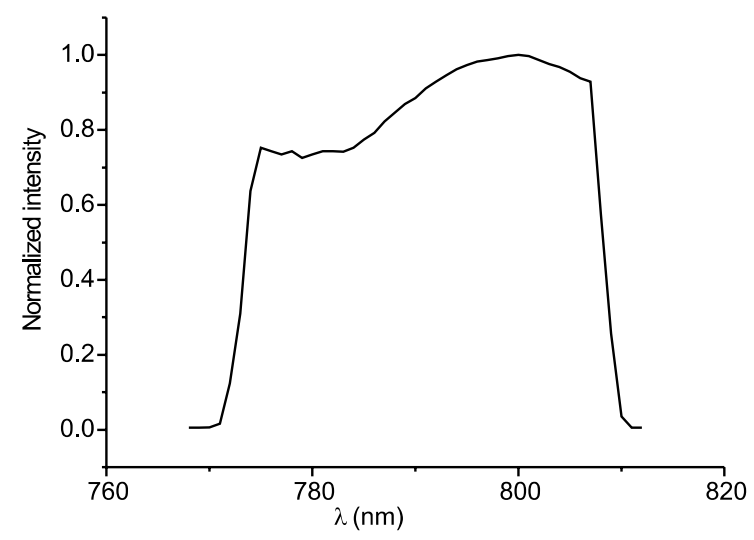

Fig. 1. Spectra of two different mode-locked regimes of operation which exist at the same parameters values and alignment condition: (a) transform-limited pulses (here named $\mathrm{P}_{1}$, see the text); (b) chirped pulses $\left(\mathrm{P}_{2}\right)$. Horizontal axis: wavelength in $\mathrm{nm}$, vertical axis: normalized intensity.

some mechanical perturbation. In other cases, the mode locking does not disappear but the normal bell shaped spectrum (Fig. 1a) switches to one with straight edges (Fig. 1b). Simultaneously, the autocorrelation indicates that the pulse is broadened, what is usually an undesirable effect. In laboratory jargon, the laser is said to have "too much glass". The shorter pulse regime is recovered by slightly removing one of the intracavity pair prisms (i.e., increasing the net negative GVD). We name the former regime of mode-locking $\mathrm{P}_{1}$, and $\mathrm{P}_{2}$ the latter. If the pulsewidth-spectrum relationship is calculated, one finds that in $\mathrm{P}_{1}$ the pulses are transform-limited, and chirped in $\mathrm{P}_{2}$. It is usually believed that the regime $\mathrm{P}_{2}$ is caused by the passage of the cavity from the negative to the positive GVD regime, which is known to produce longer, chirped pulses [9]. This passage would be caused by mechanical or thermal instabilities modifying the beam path inside the cavity. However, this informal explanation is not fully satisfactory. If one estimates the amount of beam displacement necessary for the passage from the negative to the positive GVD regime, one finds that such a displacement should be reflected in a significant deviation of the output beam, which is not observed. The laser spots of $\mathrm{P}_{1}$ and $\mathrm{P}_{2}$ slightly differ in area and shape, but not in direction. The laser often returns to the $\mathrm{P}_{1}$ regime without repositioning the prisms. In fact, it may do so spontaneously, before any change is made in the cavity.

A simple explanation of the origin of the regimes $\mathrm{P}_{1}$ and $\mathrm{P}_{2}$ is found by applying a five-variables Poincaré map description of this laser. The details of this description have been already published $[6,10,11]$, so we review only what is needed here. The pulse variables $T\left(=1 / \tau^{2}\right.$, where $\tau$ is the pulse duration) and $Q(=$ chirp $)$ in the $n+1$ round trip are related to their values in the $n$ round trip, through the discrete equations:

$T_{n+1}=\frac{T_{n}}{\left(K+I Q_{n}\right)^{2}+\left(I T_{n} / \pi\right)^{2}}$

$Q_{n+1}=\frac{\left(K+I Q_{n}\right)\left(J+L Q_{n}\right)+I L\left(T_{n} / \pi\right)^{2}}{\left(K+I Q_{n}\right)^{2}+\left(I T_{n} / \pi\right)^{2}}$

where $K, I, J, L$ are the elements of the temporal round trip gaussian matrix (they satisfy the relation $K L-J I=1$ ) which, at first order in the nonlinear index of refraction have the expressions [6]:

$K=1+2 \delta \beta^{\prime}$

$I=2 \delta$

$J=2 \delta \beta \beta^{\prime}+\beta+\beta^{\prime}$

$L=1+2 \delta \beta$

where $\delta$ is the negative value of the net GVD per round trip, and $\beta\left(\beta^{\prime}\right)$ is the nonlinear factor, or 
self-phase modulation, induced when the pulse crosses the rod going towards the output mirror (the rear mirror).

The possible modes of operation of the laser are given by the fixed points of the map, that is, the values of the variables that are equal one round trip after the other. Now, the observed multistability appears in a natural way. One of the solutions of Eqs. (1) and (2) is $T_{n}=T_{n+1}=0$, which corresponds to $\mathrm{CW}$ operation $(\tau \rightarrow \infty)$. We name this regime $\mathrm{P}_{0}$. Assume now that, based on the observations, we look for a transform-limited pulsed solution (previously named $\mathrm{P}_{1}$ ). In this case, $Q_{n}=Q_{n+1}=0$, which implies $K=L$ or $\beta=\beta^{\prime}$. This means that for $\mathrm{P}_{1}$ the nonlinear factors are nearly equal in both directions. If we ask that $Q \neq 0$ instead, $2 I Q=L-K$ and then $Q=\beta^{\prime}-\beta$. This means that the nonlinear factors are different for the two directions of propagation. The observed $\mathrm{P}_{2}$ regime corresponds to the case in which the nonlinear factor is large when the pulse propagates towards the rear mirror and negligible in the opposite direction, and hence the pulse has positive chirp. These two cases have been discussed in physical terms in Ref. [6]. Noteworthy, our approach also predicts a third mode-locking regime (we name it $\mathrm{P}_{3}$ ) for which $\beta>\beta^{\prime}$ and it has in consequence negative chirp at the output. The reason why this mode of operation has never been observed becomes clear when considering the stability regions of each solution (see Section 3 ).

The nonlinear factor $\beta$ is written as a function of the pulse variables $U$ (energy), $\sigma$ (spot radius) and $\tau$ as:

$\beta=C_{\beta} U /\left(\sigma^{2} \tau^{3}\right)$

where $C_{\beta}$ is a factor (proportional to the nonlinear index of refraction) whose precise expression is not trivial $[10,11]$. In practical terms, the numerical value of $C_{\beta}$ is unknown. Our approach is not aimed to a precise quantitative description. In a complex nonlinear system, highly sensitive to fluctuations and noise, a detailed quantitative description is almost hopeless. Instead, our approach is designed to reveal the structurally stable [12] properties of the system, as the type and relative position of the stable-unstable boundaries. In spite of these $a b$ initio limitations, our approach does provide one quantitative and structurally stable prediction that can be easily tested. From Eq. (7), the pulse duration for $\mathrm{P}_{1}$ and $\mathrm{P}_{2}$ are:

$$
\begin{aligned}
& \tau=-\delta /\left(2 \pi^{2} C_{\beta} F\right) \quad\left(\text { for } \mathrm{P}_{1}\right) \\
& \tau=-\delta /\left(\pi^{2} C_{\beta} F\right) \quad\left(\text { for } \mathrm{P}_{2}\right)
\end{aligned}
$$

where $F=U / \sigma^{2}$. Note that $\tau$ varies linearly with the net GVD, in agreement with numerical simulations and the observation. When the other three equations of the map (the ones for the energy, the beam size and radius of curvature) are taken into account, one finds that $F$ is a constant equal to the saturation energy flux multiplied by the small signal gain and the single passage feedback factor (caused by linear or passive losses) [6]. In the complete description, an extra term is added to Eqs. (8) and (9) to take into account the finite bandwidth of the amplifier [10]. Beside details, what is important here is that, provided the beam size and average power of $P_{1}$ and $P_{2}$ are roughly the same (as they are), the slope of the $\tau$ vs. GVD line is twice for $\mathrm{P}_{2}$ than for $\mathrm{P}_{1}$. This is a prediction that does not depend on the precise numerical value taken by $C_{\beta}$, and that can be easily verified experimentally.

\section{Experimental setup and observations}

A sketch of the Ti:sapphire laser we have used is shown in Fig. 2. It is constructed in the $\mathrm{X}$ configuration with a flat high reflector rear mirror $\left(\mathrm{M}_{4}\right)$, and a flat $12 \%$ output coupler. The focusing mirrors $\mathrm{M}_{2}$, and $\mathrm{M}_{3}$, have a $10 \mathrm{~cm}$ radius of curvature. The Ti:sapphire crystal is $4 \mathrm{~mm}$ long. A pair of fused silica prisms separated $60 \mathrm{~cm}$ from each other are placed inside the cavity for GVD compensation. The total length of the cavity is $172.5 \mathrm{~cm}$, resulting in a pulse repetition frequency of $86.89 \mathrm{MHz}$ and a typical average power of 400 $\mathrm{mW}$. The pump laser is a frequency doubled, diode pumped $\mathrm{Nd}: \mathrm{YVO}_{4}$ delivering $5 \mathrm{~W}$ of $\mathrm{CW}$ power at $532 \mathrm{~nm}$ and focused on the rod with a $f=10 \mathrm{~cm}$ lens. This solid state laser provides increased steadiness and repeatability, in comparison to our previous studies performed with an $\mathrm{Ar}^{+}$laser pump. The laser spectrum is measured with a 


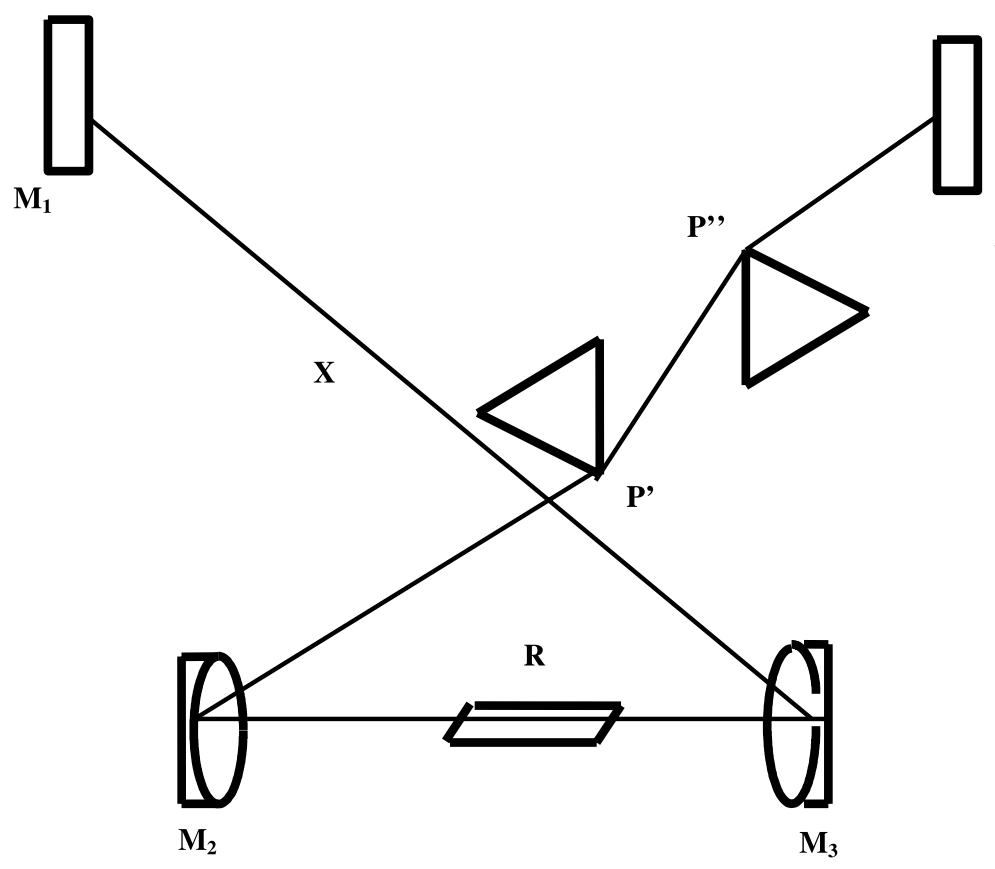

Fig. 2. Scheme of the laser cavity. $\mathrm{M}_{1}$ : output coupler, $\mathrm{M}_{2}, \mathrm{M}_{3}$ : focusing mirrors, $\mathrm{M}_{4}$ : rear mirror, $\mathrm{R}$ : Ti:sapphire rod, $\mathrm{P}^{\prime}$, $\mathrm{P}^{\prime \prime}$ : prisms for GVD compensation, $X$ : distance $\mathrm{M}_{1}-\mathrm{M}_{3}$. Prism $\mathrm{P}^{\prime}$ is mounted in a translation stage with a ruled micrometer screw, to measure the change of GVD when the prism is displaced parallel to its symmetry axis.

diode array with a resolution of $2 \mathrm{~nm}$. To measure the pulse duration, we have used an interferometric second harmonic autocorrelation technique. Because of the rapid scan scheme included in one of the autocorrelator arms it is possible to immediately detect any change of the time characteristics of the pulse by simply measuring the autocorrelation trace on the oscilloscope.

We adjust the laser to a position where $\mathrm{P}_{1}$ is the stable regime. By carefully measuring the separation between the prisms, and the beam path inside the prisms, we obtain a typical value of net GVD per round trip of about $-120 \mathrm{fs}^{2}$. At this position, mechanical noise (for example, tapping the mirrors' mounts) induces transitions to $\mathrm{P}_{2}$ and back. Then, we move one of the prisms "in", to add glass to the path and, in consequence, to reduce the absolute value of the GVD. According to the tabulated values of GVD for fused silica, advancing the prism $0.5 \mathrm{~mm}$ introduces $20.32 \mathrm{fs}^{2}$ of positive GVD (twice this value per round trip). By measuring the prism's position and the corresponding pulse duration, we measure the $\tau$ vs. $\delta$ slope. At nearly $-100 \mathrm{fs}^{2}$ the laser switches from $\mathrm{P}_{1}$ (measured pulse duration: $38 \mathrm{fs}$ FWHM) to $\mathrm{P}_{2}$ (66 fs). At this position, the noise does not produce transitions back to $\mathrm{P}_{1}$. We measure the slope for $\mathrm{P}_{2}$ in the same way as before. The measured slope for $\mathrm{P}_{2}$ is $0.61 \mathrm{fs}^{-1}$, almost exactly twice the measured value for $P_{1}, 0.32 \mathrm{fs}^{-1}$, in agreement with the predictions of the maps approach. Noteworthy, the slopes are nearly one order of magnitude larger than calculated in numerical simulations $[13,14]$, which indicates that, in practice, the nonlinear effects (which are proportional to $C_{\beta}$ ) are much smaller than expected (see Eqs. (8) and (9)). Further reduction of the absolute value of the GVD, makes (at about $-80 \mathrm{fs}^{2}$ ) the mode locking to become unstable. Eventually, the laser collapses into the $\mathrm{CW}$ regime $\left(\mathrm{P}_{0}\right)$.

One of the main advantages of the maps approach is that the stability of the solutions against 


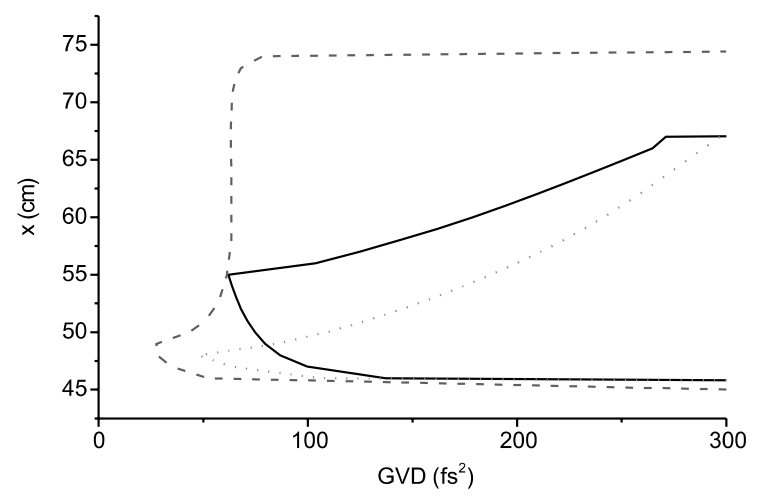

Fig. 3. Stability regions for the three mode-locking regimes. Solid line: $\mathrm{P}_{1}$, dashed line: $\mathrm{P}_{2}$, dotted line: $\mathrm{P}_{3}$. Horizontal axis: Negative net GVD inside the cavity in $\mathrm{fs}^{2}$, Vertical axis $x$ in $\mathrm{cm}$.

infinitesimal perturbations can be easily computed, by solving the eigenvalues equation of the linearized map evaluated at the fixed point. Where the moduli, in the parameters space, of one or more eigenvalues is equal to 1 , the corresponding fixed point becomes unstable. In Fig. 3, we plot the calculated regions of stability of the three predicted mode-locking solutions as a function of two parameters: the position of the output mirror $(x)$ and the net GVD. We choose these control parameters because they are easily accessible experimentally. With the help of this figure, the observed laser behavior is easily understood. For a large value of negative GVD, both $P_{1}$ and $P_{2}$ are stable and the noise induces transitions from one to the other. If glass is added to the cavity, the operation point moves leftwards, crossing the stability boundary of $\mathrm{P}_{1}$. According to the figure, the transition occurs, for $x=58 \mathrm{~cm}$, at $-150 \mathrm{fs}^{2}$ for a pulse duration (of $\mathrm{P}_{1}$ ) of 49 fs instead of the observed $-112 \mathrm{fs}^{2}$ and $\tau=37 \mathrm{fs}$. The values of $C_{\beta}$ and $F$ used to plot the figure are chosen to loosely fit the observed $\tau$ vs. $\delta$ slope (we use $0.65 \mathrm{fs}^{-1}$ for $\mathrm{P}_{2}$ ). The agreement can be improved by fine tuning of these values and $x$ but, as we already stated, the approach is not aimed to provide a numerically accurate description. What is important, in short, is that for "too much" glass added into the cavity only $\mathrm{P}_{2}$ is stable. By adding even more glass, also $\mathrm{P}_{2}$ becomes unstable and the laser collapses to the only remaining stable regime, $\mathrm{P}_{0}(\mathrm{CW})$. Note that the presented description holds, no matter the precise value taken by the parameter $x$. It is worth recalling here that the existence of a chirped solution with a larger stability region than that of the transform-limited solution was reported long ago $[15,16]$.

From the inspection of Fig. 3, two questions arise: why is the $\mathrm{P}_{3}$ regime (negatively chirped pulse) never observed? How is it possible that, in spite of the unavoidable coexistence of $\mathrm{P}_{2}$, the $\mathrm{P}_{1}$ regime is stable enough to be used in the practice? The answer to these questions lies in the numerical magnitude of the eigenvalues. Small, in moduli, eigenvalues mean that a small perturbation to the fixed point vanishes in few iterations (= round trips). Conversely, eigenvalues close to 1 mean that any perturbation makes the representative point of the system to wander, in phase space, a large number of iterations in the neighborhood of the fixed point. In consequence, the moduli of the eigenvalues are a measure of the actual ability of the fixed point to retain the system. They can be visualized as the "steepness" of the walls of the minimum of "potential" (actually, the Lyapunov surface) surrounding the stable fixed point. Even in its stable region, the eigenvalues moduli of $\mathrm{P}_{3}$ are very close to 1 and always larger than those of $\mathrm{P}_{1}$ and $\mathrm{P}_{2}$. As the stability region of $\mathrm{P}_{3}$ is fully included in the stability regions of the "steeper" fixed points $\mathrm{P}_{1}$ or $\mathrm{P}_{2}$, the system is weakly attracted to $\mathrm{P}_{3}$. In any case, it is not plausible that the system remains attached to $\mathrm{P}_{3}$ for a time long enough to be observed (what means several billions of iterations). The same argument explains why the laser prefers $P_{1}$ to $P_{2}$ in the region of large negative GVD. The eigenvalues moduli of $P_{2}$ are larger than those of $\mathrm{P}_{1}$ by a factor 2 except, of course, in the region where $\mathrm{P}_{1}$ becomes unstable. This "advantage" of $\mathrm{P}_{1}$ is due to its shorter pulsewidth, what induces a stronger nonlinearity. A stronger nonlinearity shifts the eigenvalues farther from the linear regime $\left(\mathrm{P}_{0}\right)$ which is known to have indifferent stability [17] i.e., all its eigenvalues are exactly equal to 1 , this holds in the absence of limiting apertures or bandwidth, which is an assumption of our approach. As it is shown above, this "advantage" of $\mathrm{P}_{1}$ has the drawback of a smaller stability region. 
Finally, it is worth mentioning that the stableunstable transition for increasing GVD, of both $\mathrm{P}_{1}$ and $\mathrm{P}_{2}$, are caused by the same eigenvalue crossing -1 . The related eigenvector is practically collinear with the variable "spot area". This means that, by adding glass to the cavity, we should observe a period-doubling bifurcation in the spot size, with negligible fluctuations of the other pulse variables. This is the bifurcation previously named " $2 \mathrm{~A}$ " which was, indeed, observed in a previous work [6].

\section{Summary}

We have shown that the observed bistability between two mode-locking regimes in KLM Ti:sapphire lasers is not caused by a change in the sign of the net GVD, but that it is an intrinsic property of the system in the negative GVD region. The reason the system operates in one or the other regime is evident from the shape and size of their stability regions obtained from a five-variables Poincaré map approach, and from the relative moduli of their eigenvalues. We have also experimentally verified the prediction that the $\tau$ vs. $\delta$ slope is twice as large for the chirped regime as for the transform-limited one. In previous observations, we had also verified that the mode-locking regime loses stability (when increasing GVD) through a period-doubling bifurcation that affects mainly the spot size variable. In conclusion, the Ti:sapphire KLM laser displays a variety of interesting nonlinear dynamics, which essential features can be understood in simple terms with the approach using a Poincaré map.

\section{Acknowledgements}

This work was supported by the projects PIP CONICET 425/98 and 639/98. Thanks to Prof. Oscar E. Martínez for his remarks on the use of the interferometric autocorrelator.

\section{References}

[1] D. Spence, P. Kean, W. Sibbett, 60-fs pulse generation from a self-mode-locked Ti:sapphire laser, Opt. Lett. 16 (1991) 42.

[2] J.F. Cormier, M. Piché, F. Salin, Suppression of beam breakup in self-mode-locked Ti:sapphire lasers, Opt. Lett. 19 (1994) 1225.

[3] Q. Xing, W. Zhang, K.M. Yoo, Self Q-switched self modelocked Ti:sapphire laser, Opt. Commun. 119 (1995) 113.

[4] M. Kovalsky, A. Hnilo, C. González Inchauspe, Hidden instabilities in the Ti:sapphire KLM laser, Opt. Lett. 24 (1999) 1638.

[5] D. Cote, H.M. van Driel, Period doubling of a femtosecond Ti:sapphire laser by total mode locking, Opt. Lett. 23 (1998) 715 .

[6] M. Kovalsky, A. Hnilo, Stability and bifurcations in KLM Ti:sapphire lasers, Opt. Commun. 186 (2000) 155.

[7] J. Jasapara, W. Rudolph, V. Kalashnikov, D. Krimer, I. Poloyko, M. Lenzner, Automodulations in Kerr lens mode locked solid states lasers, J. Opt. Soc. Am. B 17 (2000) 319.

[8] I.P. Christov, V.D. Stoev, M.M. Murnane, H.C. Kapteyn, Mode locking with a compensated space-time astigmatism, Opt. Lett. 20 (1995) 2111.

[9] H.A. Haus, J.G. Fujimoto, E.P. Ippen, Structures for additive pulse mode locking, J. Opt. Soc. Am. B 8 (1991) 2068.

[10] A. Hnilo, KLM Ti:sapphire laser description with an iterative map, J. Opt. Soc. Am. B 12 (1995) 718.

[11] M.A. Marioni, A. Hnilo, Self-starting of self-mode-locking Ti:sapphire lasers. Description with a Poincare map, Opt. Commun. 147 (1998) 89.

[12] P.T. Saunders, An Introduction to Catasprophe Theory, Cambridge Press, New York, 1980.

[13] T. Brabec, Ch. Spielmann, F. Krausz, Mode locking in solitary lasers, Opt. Lett. 16 (1991) 1961.

[14] J.L.A. Chilla, O.E. Martinez, Space-temporal analysis of the self mode locked Ti:sapphire laser, J. Opt. Soc. Am. B 10 (1993) 638.

[15] M. Murnane, H. Kapetyn, J. Zhov, C. Huang, D. Garvey, M. Asaki, Advances in fs pulse generation through the use of solid state lasers; OSA Annual Meeting 1993, Invited Communication WD1.

[16] V. Petrov, D. Georgiev, J. Herrmann, U. Stamm, Theory of CW passive mode locking of solid state lasers with addition of nonlinear index and GVD, Opt. Commun. 91 (1992) 123.

[17] L.M. Sanchez, A. Hnilo, Optical cavities as iterative maps in the complex plane, Opt. Commun. 166 (1999) 229. 